$\mathbb{T}$ periodica polytechnica

Civil Engineering

$58 / 2(2014) 131-136$

doi: $10.3311 /$ PPci.7489

http://periodicapolytechnica.org/ci

Creative Commons Attribution (1)

RESEARCH ARTICLE

\section{Variations of the gravity field due to excavations of the Budapest Metro4 subway line}

\author{
Csaba Égető / Nikolett Rehány / Lóránt Földváry
}

Received 2013-04-25, revised 2013-06-10, accepted 2013-07-11

\begin{abstract}
The effect of the construction of the 4th subway line of Budapest (Metro4) on the potential surfaces of the gravity field has been investigated from the aspects of monitoring vertical deformation. In the study mass loss due to the excavation of the two tunnels and of the stations has been considered. Practically, the effect of the mass loss on leveling measurements was determined at a level $1 \mathrm{~m}$ above the ground, roughly simulating common instrument heights. The indirect effect of the actual deformations of the physical surface on the leveling was not considered, so in the investigation a rigid Earth has been assumed. In the study, different arrangements of the leveling lines and of the excavations were examined, furthermore, the steepness of the leveling line and the density of the leveling points were analyzed. According to the results, under certain arrangements of the leveling line, the effect can reach the $0.05 \mathrm{~mm}$ order of magnitude, which is equivalent to the accuracy of the precise leveling.
\end{abstract}

\section{Keywords}

temporal variations of gravity $\cdot$ deformation of potential surfaces $\cdot$ precise leveling $\cdot$ virtual veritcal motion $\cdot$ prism modelling

\section{Csaba Égető}

Department of Geodesy and Surveying, Budapest University of Technology and Economics, Múegyetem rkp. 3., H-1111 Budapest, Hungary

e-mail: ecsaba@agt.bme.hu

\section{Nikolett Rehány}

Faculty of Civil Engineering, Budapest University of Technology and Economics, Múegyetem rkp. 3., H-1111 Budapest, Hungary

\section{Lóránt Földváry}

Department of Geodesy and Surveying, Budapest University of Technology and Economics, Múegyetem rkp. 3., H-1111 Budapest, Hungary

\section{Introduction}

Temporal variations of gravity field are of central interest in geodesy and geophysics, since it reflects the mass redistributions on the surface and within the planet Earth, providing a unique tool for investigating its interior [14], [8]. Nowadays there is an urge of such analyses in global and regional scales due to the obvious signs of a climate change [11]. The state-of-theart gravity satellites turn to be very efficient tools for that [9], [16], [12], [10]. As the knowledge of the temporal gravity in global and regional scales improves notably, there is a demand for the local gravity variations as well, since satellites are insensitive for the temporal varying gravity in local scales. Thus, it can only be determined independently by terrestrial measurement techniques e.g. [3] to provide the short frequency part of the spatial wavelength spectrum [15].

In the present study temporal variation of a local gravity feature due to a technogenic mass redistribution effect is modeled and analyzed. In case of a huge industrial project, notable redistribution of soil occurs due to the earthworks. It does affect the gravity field [1]. As so, it also has an effect on the local horizontal and vertical directions. As surveying instruments are set up with respect to the horizontal or vertical, it does affect the accompanying surveying measurements. In the present study certain geodetic aspects of an actual surveying engineering application is considered. The excavation of various layers of soil during the construction of the new subway line of Budapest, Metro4 is investigated from the aspect of its effect on the simultaneously performed vertical deformation measurements.

\section{Theoretical Background and Formulation}

\subsection{The classical formulae of modelling}

In case of excavating tunnels, deformations on the surface are expected to be accompanied. Furthermore, the removal of a notable amount of soil changes the structure of the gravity field too. Therefore, observed height variation, $d H$ consists of two components [1], [13]:

$$
d h=d H+d N
$$


where $d h$ refers to the actual surface deformation, and $d N$ is change of the geoid undulation due to the mass loss. (Generally, in this study the differential symbol, $d$ refers to change in time and not in space.) The change of the geoid undulation does affect the height measurements, but has no effect on the neighboring structures, buildings. Such a height difference involved to the geometrical leveling is rather the consequence of theoretical looseness than actual vertical displacement, thus the confusion of the two should be avoided.

Change of the geoid undulation by time, $d N$ in Eq. (1) is usually interpreted as (cf. [2] and [17]):

$$
d N=\frac{R}{4 \pi g} \iint d g \cdot S(\psi) d \sigma+\frac{R}{4 \pi g} \iint \frac{2 g}{R} d h \cdot S(\psi) d \sigma
$$

The terms on the right hand side of Eq. (2) refers to two independent sources of mass variations:

1 deformation of the equipotential surface due to the mass loss corresponding to the tunnelling,

2 deformation of the equipotential surface due to the actual vertical displacement of the physical surface.

For every infinitely small area, $d \sigma=\cos \varphi d \varphi d \lambda$ the spherical distance from the point of interest, $P$ is computed as

$$
\cos \psi=\cos \theta \cos \theta_{P}+\sin \theta \sin \theta_{P} \cos \left(\lambda-\lambda_{P}\right)
$$

Eq. (2) describes the temporal variation of geoid undulation as a function of that of the gravity anomaly, $d g$, weighted by the Stokes function, $S(\psi)$. Considering a mass anomaly model, $d m$ as known input data, Eq. 22 can be solved by using the Newtonian gravitational law,

$$
d g=k \int \frac{d m}{r^{2}}
$$

A more direct approach to obtain temporal variations of geoid undulation from a mass variation model is to derive geoid undulation variations from Newtonian potential using the Bruns' equation.

$$
d N=\frac{1}{\tilde{\gamma}} k \int \frac{d m}{r}
$$

In Eq. (5) $\tilde{\gamma}$ refers to the mean normal gravity between the point and the reference ellipsoid along the plumb line. Note that Eq. (5) is analogue with the first term of the right hand side of Eq. (2).

In the followings a single height difference between stations $A$ and $B$ with repeated levelling measurements performed at time $t$ and $t_{1}$ is considered (c.f. Fig. 1). Temporal variation of geoid undulation between the two stations can be defined as the difference of the temporal changes in each point

$$
d N_{A B}=d N_{B}\left(t_{1}-t_{0}\right)-d N_{A}\left(t_{1}-t_{0}\right)
$$

The measured vertical displacement is interpreted as temporal change of the height difference

$$
d H_{A B}=H_{A B}\left(t_{1}\right)-H_{A B}\left(t_{0}\right)
$$

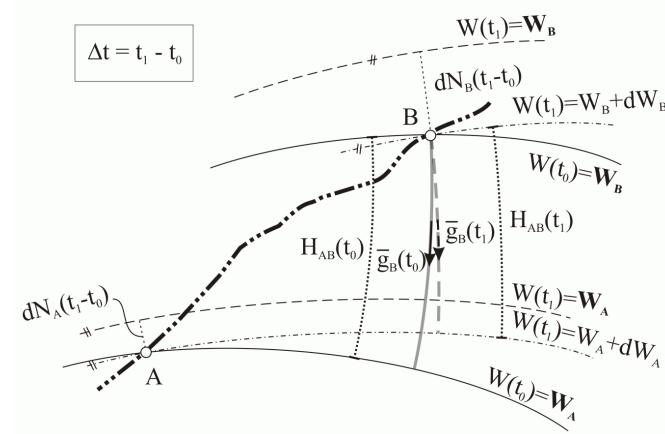

Fig. 1. Visualization of the apperent vertical displacements due to the temporal variation of gravity field

Then the relationship between change of geoid undulation and observed vertical displacement can be derived from equations above as follows (c.f. equation 212.5 of [1], on page 31):

$$
d H_{A B}=-d N_{A B}-\frac{\tilde{g}_{B}\left(t_{1}\right)-\tilde{g}_{B}\left(t_{0}\right)}{\tilde{g}_{B}\left(t_{1}\right)} H_{A B}\left(t_{0}\right) .
$$

In Eq. (8) $\tilde{g}_{B}$ refers to the mean gravity between levels of $A$ and $B$ along the plumb line through point $B$. This is approximately equal to the gravity at the half of the $H_{A B}$ height difference.

\subsection{Elaborating the formulation}

In the present case, height of the benchmarks on the test region is available after the tunneling, after the excavation has been performed. However, according to [1], $H_{A B}\left(t_{0}\right)$ in Eq. (8) refers to the original height difference, before the deformation takes place. As so, a modified version of Eq. (8) is derived:

$$
d H_{A B}=-d N_{A B}-\frac{\tilde{g}_{B}\left(t_{1}\right)-\tilde{g}_{B}\left(t_{0}\right)}{\tilde{g}_{B}\left(t_{1}\right)}\left(H_{A B}\left(t_{1}\right)+d N_{A B}\right)
$$

For details of the derivation see [5].

In the present study effect of the excavation on the level surfaces was estimated based on equations Eq. (5) - Eq. (9)). The integral of equation Eq. (5) was approximated by a summation over mass elements. A preceding study has already been presented by [4], where the non-deformational effects due to the tunneling were investigated in a regular grid, and not along leveling lines as it is to be presented here. In this study the effect of the actual surface deformation on the gravity field, i.e. second term of the right hand side of Eq. (2) has been neglected. The effect due to actual surface deformations has been analyzed and discussed by [6].

\section{Data and Calculations}

The calculations have made use of the following available data:

1 longitudinal sections and cross sections of the tunnels provided by the DBR Metro Project Directorate

2 soil density along the tunnels provided by the Geovil Ltd

3 gravity anomaly data in the vicinity of the tunnels provided by the Hungarian Geological and Geophysical Institute 
4 horizontal trace of the leveling lines provided by the Consortium of the Soldata Ltd and Hungeod Ltd

The top view of the tunnels and the location of the surrounding gravimetric data are displayed on Fig. 22 The used coordinates are the local stereographic coordinates (BÖV, equivalent to Budapest Municipal Projection system).

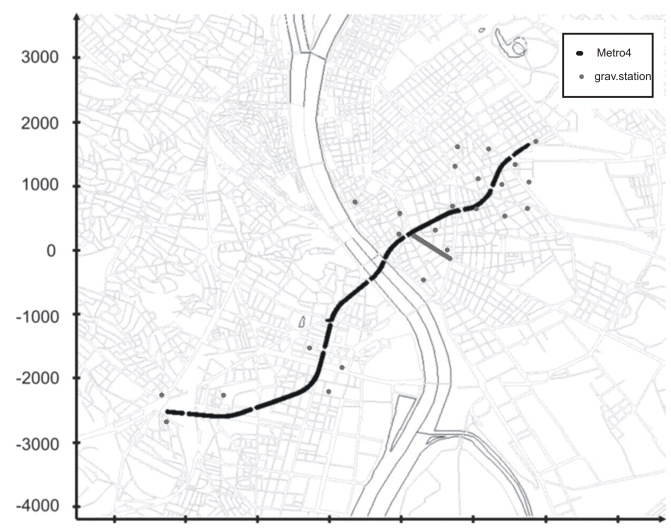

Fig. 2. The tunnel (solid black line interrupted at the stations) and the gravimetric points (grey stars) with main structures of Budapest in the background. The axis are local stereographic coordinates in $\mathrm{m}$.

First a 3D geometry model of the tunnels and of the stations was determined based on the longitudinal sections. The corresponding mass model of the excavated soil has been derived by using the soil density data, and the effect of the excavation on geoid undulation was determined by Eq. (5). The resolution of the mass model was $30 \mathrm{~cm}$, i.e. the masses were concentrated into cubes with $30 \mathrm{~cm}$ long edges. Then the adequate formulas of rectangular prisms (c.f. [7]) were simplified to simple point masses, and Eq. (5) was used. The accuracy loss due to this simplification was verified by [4], and it was found to cause negligible difference. In case of the stations, the exact rectangular prism formula was used. The mass removals due to excavation in the tunnels and in the stations were added, and the result is considered to be an estimate of the effect of the excavation without any displacements assumed to occur.

Subsequently, gravity value was interpolated from the neighboring gravity data to each point of evaluation, and for every single height differences of the leveling line the effect of the tunneling on the every single height measurements was determined by Eq. (8).

The effect of the excavation was evaluated at the points of actual vertical control leveling lines on the south-east part of the subway line, c.f. Fig. 3 (note that the interruptions of the tunnels refer to the presence of stations). To the known heights of the benchmarks $1 \mathrm{~m}$ has been added to simulate a typical instrument height. Altogether there were 40 leveling lines involved in the analysis. Among them, only some typical features are to be presented in the following section.

\section{Results}

Generally, the effect of the excavation on the measurements along the leveling lines is varying between 0 and $0.01 \mathrm{~mm}$.
Though it does not seem to be much, it is important to note that effect of this theoretical error is in the same magnitude than that of the precision of the precise level instruments. As so, it is detected in the measurements. Furthermore, it could be detected that under certain conditions it accumulates along the leveling line. The accumulation of the error depends on the orientation of the line, the distance of the instrument and the staff, and the height difference.

\subsection{Dependence on the relative orientation}

The orientation of the leveling line relatively to the orientation of the tunnels makes notable impact on the result. The two extremes of the orientation is when the tunnel and the leveling line are parallel to each other and when they are perpendicularly cross each other. There was no good example for the perpendicular crossing (c.f. Fig. 3), but as an example, a nice example for that is adopted from [4] on a different part of the same subway line. The perpendicular crossing with very short distances between the instrument and the staff is displayed in Fig. 4. The figure shows the longitudinal section of the leveling line. The ordinate is the distance from the midpoint of the two tunnels, and the abscissa is the estimated error in $\mu \mathrm{m}$. The horizontal location of the tunnels is displayed by the two circles close to the zero value of the ordinate. From the figure at the crossing a sudden change of sign can obviously be detected. Due to that, when the line is processed, the different sign of the error neutralizes the effect. So practically the closure error is compensated.

On the other hand, in cases, when the leveling line runs parallel to the tunnels, the error effect becomes similar for every single height differences. Thus the error is accumulating along the line. For such an example in Fig. 5 and Fig. 6 the line no. 34 is presented. Fig. 5 shows the horizontal arrangement of the line, which is parallel to the tunnels, passing through at the upper left corner. The abscissa and the ordinate refer to the BÖV coordinates in meter. On Fig.6 the error estimate is shown along with the longitudinal section of the leveling line. The zero ordinate refers to the starting point. The error on the abscissa is displayed in $\mu \mathrm{m}$.

As it can be seen, the error has consequently the same sign, affecting all the measurements in the same extent. On Fig.6 also the back and forth errors are displayed. It is obvious that this error cannot be eliminated by averaging back- and forth measurements. As so, in such a case the error remains in the adjusted heights even in case of a thorough processing.

\subsection{Dependence on the height difference}

In the cases of the tested leveling lines, with the exception of line no.14, the height difference between the first and the last point is only $1-2 \mathrm{~m}$. The last section of the line no. 14 climbs up to a hill, resulting in a $13 \mathrm{~m}$ height difference along the line, cf. Fig. 7 and Fig. 8, On Fig. 7 and Fig. 8 the axes are the same as in the case of Fig. 5 and Fig. 6, respectively.

According to Fig. 8, the dependence of the error on the height 


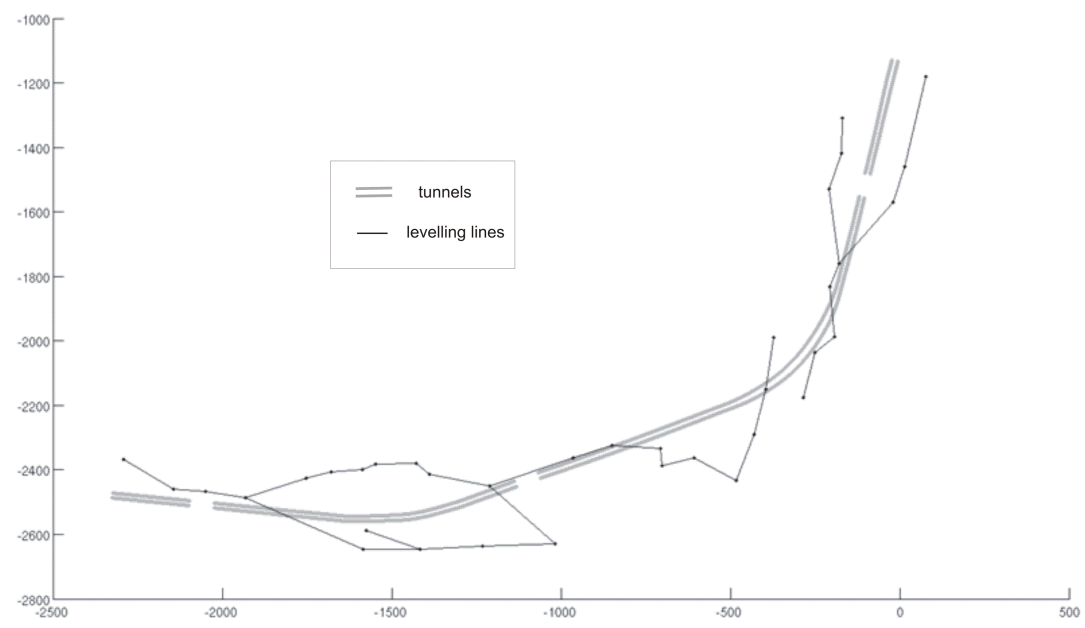

Fig. 3. The location of the vertical control lines (thin lines) with respect to the tunnels (thick parallel lines).

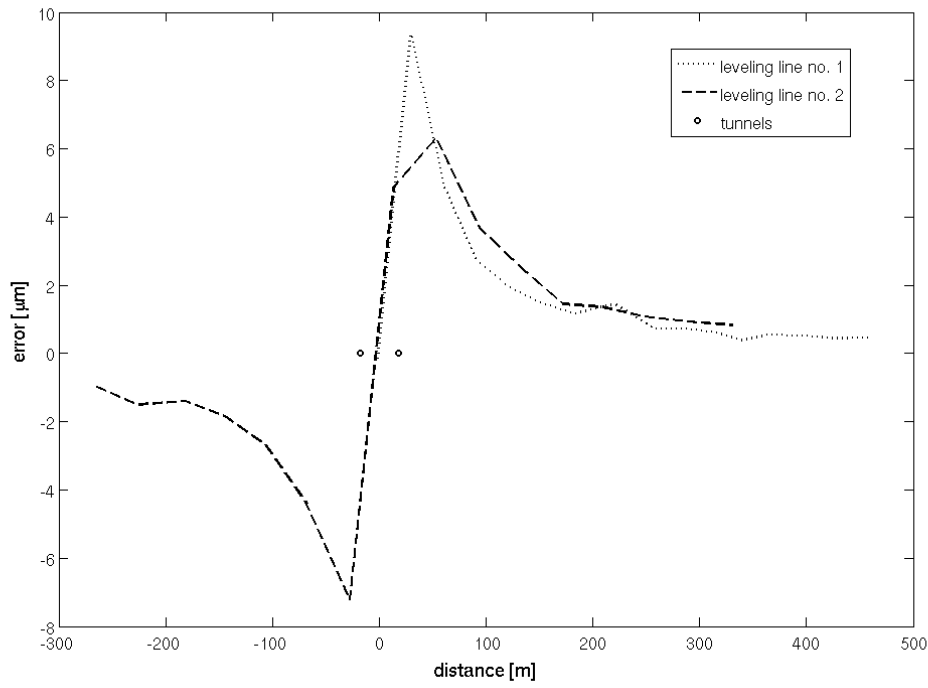

Fig. 4. An example on the effect of perpendicularly crossing the tunnels. 


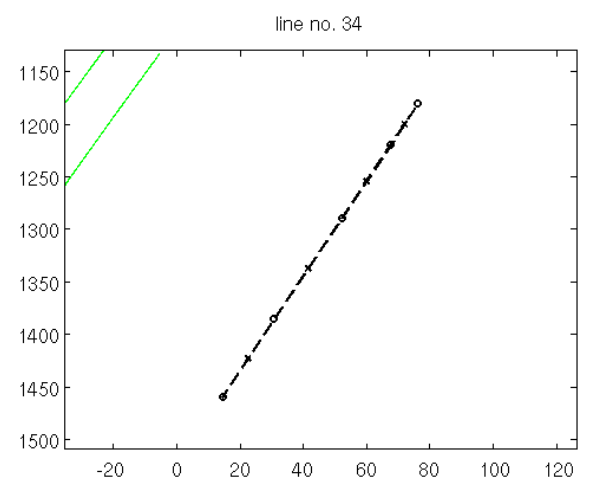

Fig. 5. Top view of the levelling line no. 34. dashed line: leveling line. symbol 'o': place of staff, symbol ' $x$ ': assumed place of the instrument. The two tunnels are marked by thin lines on the upper left part of the figure.

difference is obvious. Where the steep part comes, the error suddenly increases with an order of magnitude. The result is not surprising at all, as in Eq. (9) height difference appears as a multiplier.

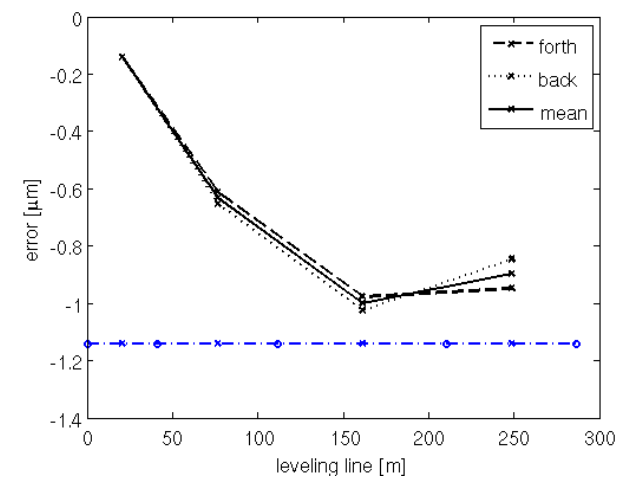

Fig. 6. The estimated error for every single height differences along levelling line no. $34 \mathrm{in} \mu \mathrm{m}$. The error along the back- and forth levelling is determined independently, then the mean error was derived. At the bottom staff- and instrument places are shown in the same manner as in Fig. 5

\subsection{Dependence on instrument-staff distance}

Oddly, the dependence of the error on the distance of the level instrument and the leveling staff cannot be demonstrated from real measured data, like in the present case. Although it is obvious: measuring with short distances means a finer resolution of the level surfaces, than long ones. Thus with shorter distances the error can be decreased.

The reason it could not be demonstrated is a very practical one: in case of an actual work, short distances are used only in case of large height differences. So it is used for steep fields, such as line no 14 on Fig. 6 However, in this case the unlike effect of the height difference suppresses the effect of the short distances.

\section{Conclusions}

Basically, the effect of the excavation on the measurements along the leveling lines was found to have theoretical importance in most cases. Still, in such cases, when the leveling line

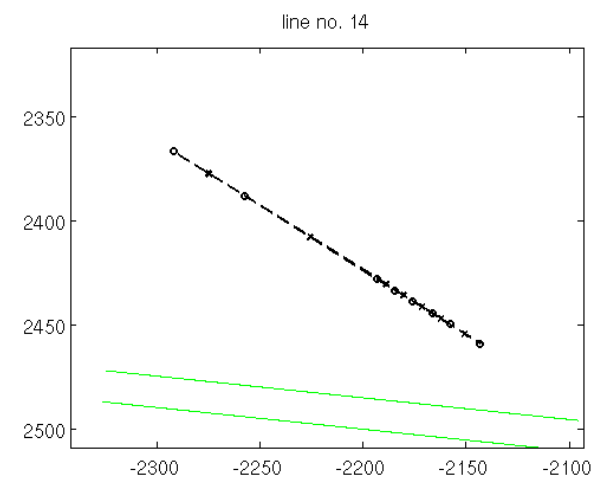

Fig. 7. Top view of the levelling line no. 14. dashed line: leveling line. symbol 'o': place of staff, symbol ' $x$ ': assumed place of the instrument. The two tunnels are marked by thin lines on the bottom of the figure.

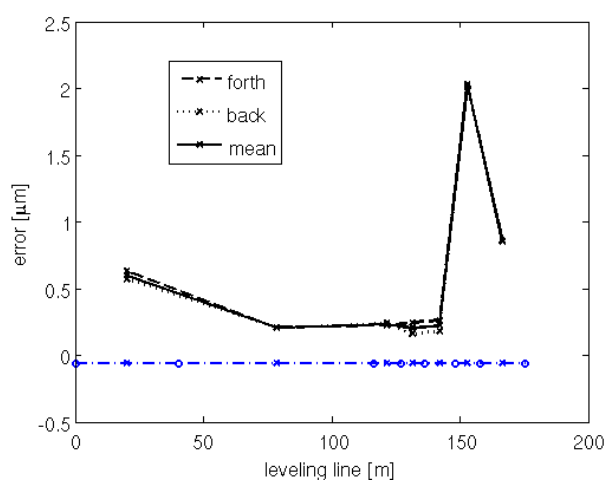

Fig. 8. The estimated error for every single height differences along levelling line no. 34 demonstrating a very steep levelling line at its final section. The error along the back- and forth levelling is determined independently, then the mean error was derived. At the bottom staff- and instrument places are shown in the same manner as in Fig. 7 
was running parallel to the tunnels, the cumulative error reached the precision of precise leveling. Even though precise leveling measurements are subsequently processed, dropping the random errors out, in case of parallel orientation the error remains in the adjusted results and becomes systematic.

Further relevance of the study is that Metro4 is just a subway line. There are several other constructions resulting in huge excavations, removal of notable masses. It is important to keep in mind that redistribution of mass does affect the gravity field. In case of a large construction there is always a demand for surveying control measurements in order to detect actual deformations. Thus it is worth to conclude that surveying control measurements are infected by the temporal variation of the gravity field as well, and that the two cannot be separated from the measurement, only by theoretical considerations. It remains the case as long surveying methods make use of the gravity field for adjusting the direction of the horizontal and the vertical.

Finally, it is worth to note that this study was considering apparent deformations only. In reality, there are always real deformations as well. The actual deformations occur much closer to the instrument than the tunnels are, it is just $1-1.5 \mathrm{~m}$ below the instrument. These deformations also have their impact on the gravity field. It means that real deformations have two effects on the control measurements: a direct effect, as they are the deformations to be detected, and an indirect effect, as they alter the gravity field. Even though these close-to-instrument deformations can be small in magnitude, as the gravity vanishes with square distance, they can affect the measurements in larger extent. As a complementary research to the present study, it is essential to quantify the magnitude of the indirect effect of real deformations.

\section{References}

1 Biró P, Time variation of height and gravity, Wichmann Verlag; Karlsruhe, 1983.

2 Biró P, Thong NC, Weisz E, Modelling of secular variations in gravity and in geoidal undulations, Periodica Polytechnica Separatum, 30(1-2), (1986), 23-26.

3 Csapó G, Laky S, Égető C, Ultmann Z, Tóth G, Völgyesi L, Test measurements by Eötvös torsion balance and gravimeters, Periodica Polytechnica Civil Engineering, 53(2), (2009), 75-80, DOI 10.3311/pp.ci.2009-2.03

4 Égető C, Földváry L, The effect of the Metro 4 tunnel system on the gravity field (in Hungarian), Geomatika, XIV(1), (2011), 17-26.

5 Égető C, Földváry L, Numerical accuracy analysis of modeling excavation induced gravity field variations, Proceedings in Global Virtual Conference: The 1st International Global Virtual Conference, 1(1), (2013), 549-554.

6 Égető C, Földváry L, Huszák T, The effect of tunnelling on repeated precise levelling measurements for vertical deformation control of the Metro4 project, Journal of Geodetic Science, 3(2), (2013), 95-102.

7 Holstein H, Gravimagnetic anomaly formulas for polyhedra of spatially linear media, Geophysics, 68(1), (2003), 157-167, DOI 10.1190/1.1543203

8 Paterson NR, Reeves CV, Applications of gravity and magnetic surveys:The state-of-the-art in 1985, Geophysics, 50(1), (1985), 2558-2594, DOI $10.1190 / 1.1441884$

9 Rummel R, Balmino G, Johannessen J, Visser P, Woodworth P, Ded- icated gravity field missions - principles and aims, Journal of Geodynamics, 33(1), (2002), 3-20, DOI 10.1016/S0264-3707(01)00050-3

10 Schmidt M, Fengler M, Mayer-Gürr T, Eicker A, Kusche J, Sanchez L, Han S-C, Regional gravity modeling in terms of spherical base functions, Journal of Geodesy, 81(1), (2008), 17-38, DOI 10.1007/s00190-006-0101-5

11 Sideris MG (ed.), Observing our Changing Earth, Series: International Association of Geodesy Symposia, Vol. 133, Springer Verlag, 2008, DOI 10.1007/978-3-540-85426-5

12 Somodi B, Földváry L, Application of numerical integration techniques for orbit determination of state-of-the-art LEO satellites, Periodica Polytechnica Civil Engineering, 55(2), (2011), 99-106, DOI 10.3311/pp.ci.2011-2.02

13 Thong NC, Determination of true surface movement with spherical harmonic expansion of the variation in geopotential (in Hungarian), Geodézia és Kartográfia, 37(4), (1985), 261-265.

14 Torge W, Geodesy, Valter de Gruyten; Berlin, New York, 2001, DOI $10.1515 / 9783110879957$

15 Szücs E, Validation of GOCE time-wise gravity field models using GPSlevelling, gravity, vertical deflections and gravity gradient measurements in Hungary, Periodica Polytechnica Civil Engineering, 56(1), (2012), 3-11, DOI 10.3311/pp.ci.2012-1.01

16 Wahr J, Swenson S, Zlotnicki V, Velicogna I, Time-variable gravity from GRACE: First results., Geophysical Research Letters, 31, (2004), 01-04, DOI 10.1029/2004GL019779

17 Weiss E, Modelling of true vertical surface movements (in Hungarian), Geodézia és Kartográfia, 37(2), (1985), 101-105. 